\title{
GAMMA-RAY BURSTS: THEORETICAL CONSIDERATIONS
}

\author{
P. MÉSZÁROS \\ Pennsylvania State University, \\ 525 Davey Lab, University Park, PA 16802, USA
}

\section{Introduction}

The discovery of the afterglows of gamma-ray bursts (GRB) at X-ray, optical and radio wavelengths heralds a new era in the investigation of these objects. This provides a quantum jump in the type of observational information available, providing much more severe constraints on models, and probably represents the light at the end of the tunnel for this long-standing puzzle of astrophysics.

The first major development in this respect was that the overall features of the first GRB detected over time scales $\gtrsim$ days at X-ray (X) and optical $(\mathrm{O})$ wavelengths, GRB 970228 (Costa 1997 ) agreed quite well with theoretical expectations from the simplest relativistic fireball afterglow models published in advance of the observations (Mészáros \& Rees, 1997a); see also (Vietri, 1997a). A number of theoretical papers were stimulated by this and subsequent observations (e.g. Tavani, 1997; Waxman, 1997a; Reichart, 1997; Wijers et al., 1997 among others), and interest has continued to grow as new observations provided apparently controversial evidence for the distance scale and the possible host (Sahu et al, 1997). New evidence was added when the optical counterpart to the second discovered afterglow (GRB 970508) yielded a redshift lower limit placing it at a clearly cosmological distance (Metzger et al., 1997), and this was strengthened by the detection of a radio counterpart (Frail et al, 1997; Taylor et al, 1997) as well as evidence for the constancy of the associated diffuse source and continued power law decay of the point source (Fruchter, et al, 1997).

This new evidence reinforces the conclusions from previous work on the spatial distribution of bursts in the sky, which indicate that they are highly isotropic and compatible with a cosmological distribution (e.g Fishman, these proceedings). At the same time, investigations of the physics of fireball models of GRB have continued to probe the $\gamma$-ray behavior of these objects. Observational material on this is provided chiefly by a superb data base of over 1700 bursts accumulated in the BATSE 4B catalog, complemented by data from the OSSE and Comptel instruments on CGRO as well as Ulysses, KONUS and other experiments. Much of the recent theoretical work has concentrated on modeling the time structure expected from internal and external shock models, and the spectra ard spectral-temporal correlations.

\section{Afterglows: A Breakthrough}

The breakthrough Beppo-SAX observation of GRB 970228 provided both a study of the long-term $\mathrm{X}$-ray decay (hours to days) and an accurate localization permitting subsequent optical follow-ups (extending to months). The X-ray and optical sources are both point-like, as expected for a fireball at cosmological distances (dimension $\sim 0.1-1 \mathrm{pc}$ after $\sim$ months). The spectrum is a power law, as expected from synchrotron or inverse Compton (IC) from shock-accelerated non-thermal electrons in a decelerating shell interacting with an external medium ((Mészáros \& Rees, 1997a)), and as predicted, it decays as power law in time with an index close to the expected value (see also Vietri, 1997a). Furthermore, a fuzzy extended source was identified around the point source. While the initial optical magnitude of the point source decayed from $\sim 20$ to $\sim 24$, there was uncertainty as to whether the diffuse source remained constant and whether it showed any proper motion. However, the September 1997 HST images (Fruchter et al, 1997) have dispelled doubts, indicating 
that the diffuse source remained at $m_{R} \sim 25.5$ with negligible proper motion, being compatible with a distant $(z \sim 1)$, faint (possibly irregular) galaxy, while the optical point source is still present at $m_{R} \sim 28$, right along the extrapolation of the earlier power law.

Another highlight was the detection of the afterglow of GRB 970508, which largely followed the pattern of GRB 970228, but which added considerable excitement because of new, even if not entirely unexpected, features. One of these is that the optical flux initially rose as a power law, followed by a decay similar to the earlier case. This, in fact, is a prediction of the very simplest expanding cloud models where the spectrum has a peak which initially is at energies higher than optical (Katz, 1994b), and is in agreement with the Mészáros \& Rees (1997a) simplest model. Another is the detection of a radio afterglow in GRB 970508, about a week after the outburst, peaking after weeks and then decaying slowly. With a self-absorption frequency around 5-10 GHz (overestimated in early calculations), this is also compatible with the 'simplest' model (e.g. Waxman, 1997b, Katz \& Piran, 1997). Furthermore, scintillations in the radio spectrum, predicted by Goodman (1997), were also observed (Frail et al, 1997), providing a nice double-check on the physical dimension of the source of $\sim 0.1 \mathrm{pc}$. An interesting, and less expected result is that the scintillation is of large amplitude and broad band, suggesting it is diffractive (Waxman, etal, 1997). This requires a small size, which comes from the fact that the intensity is concentrated in a ring of radial extent substantially smaller than the radius of the visible disk (Waxman, 1997, Panaitescu \& Mészáros , 1997). This is because for equal observer times one sees an egg-shaped region of the outflow and the portion around the edges corresponds to a younger, hence hotter and higher field, stage of the remnant. Another unexpected feature is that the optical light curve may have been steady or decaying for a brief (few days) initial period before it started to rise and then decay (Pedersen, 1997). One explanation for an initial decay could be that it is due to emission from a central jet, which later becomes overwhelmed by emission from a more energetic isotropic outflow at larger angles (Mészáros, et al, 1997).

A question is why some bursts (e.g. GRB 970111) are detected in $\gamma$-rays but not in $\mathrm{X} / \mathrm{O}$, despite being in in the field of view of Beppo-SAX. One reason may be that the $\gamma$-ray emission could be due to internal shocks (leaving essentially no afterglows, (Mészáros \& Rees, 1997a)), and the environment has a very low density, so the external shock occurs at larger radii and over longer times than in "canonical" afterglows, resulting in a sub-threshold X-ray intensity. This may be the case if GRB arise from compact binaries which are ejected to considerable distances from the host galaxy, where the external density may be much lower than the typical ISM values. Low density environments may also occur if the GRB goes off inside a pulsar cavity inflated by one of the precursors in the binary. This give rise to a deceleration shock months after the GRB with a much lower brightness. Conversely, an interesting consequence of anisotropic models (Wijers et al, 1997; Rhoads, 1997; Mészáros , et al 1997) is that there could be a large fraction of detectable afterglows for which no $\gamma$-rays are detected. The outflow at large angles is certain to be more baryon-loaded, and therefore of lower $\Gamma$, so that the shocks would occur later and would be at longer wavelengths.

It is also possible that some bursts arise in unusually high density environments (such as a starforming region, where failed supernova or hypernova progenitors may reside (Paczyński, 1997). This could lead to a more rapid onset of the deceleration leading to the X-ray phase, and it would also imply an increased neutral gas column density and optical depth in front of the source. A special case is that of GRB 970828 , where $\mathrm{X}$ rays have been observed, but no optical radiation down to faint levels (Groot et al. 1997). The presence of a significant column density of absorbing material has been inferred from the low energy turnover of the X-ray spectrum (Murakami et al., 1997), and the corresponding dust absorption may in fact be sufficient to cause the absence of optical emission (Wijers \& Paczyński, private comm.). The difference between the low density and high density environments cases could be tested if future observations of afterglows reveal a correlation with the degree of galaxy clustering or with individual galaxies. 


\section{Fireball and Shock Physics}

The generic dissipative fireball model is a fairly robust astrophysical scenario, independent of the particular type of progenitor, based only on the fact that it must inject the required large amount of energy inside the inferred small volumes in the small timescales characteristic of GRB. The microphysics and plasma physics involved are standard, and have been used extensively in other astrophysical situations. The cosmological shock-fireball model appears to have received strong confirmation from the afterglow observations, and from the fact that many of the basic gamma-ray signatures can be understood within the framework of the model without undue parameter twisting. The basic ingredients (a high $\Gamma$ outflow, collisionless shocks, magnetic field generation at some fraction of equipartition, acceleration of electrons to a power law, efficient energy exchange between protons and electrons) are standard problems (or standard fare) in AGN, supernova remnants and other high energy sources. In AGN, in particular, conditions qualitatively similar seem to obtain, and these sources are known to have in many cases efficiencies of at least tens of percent in converting bulk kinetic energy into fields, nonthermal particles and radiation. As in those sources, for GRB fireballs it is assumed that the fluid approximation is valid whenever the usual plasma kinetic theory criteria are satisfied, e.g. that the dimensions of the region are much larger than the proton gyroradius or the proton Debye length. The shocks serve to reconvert the kinetic energy of the outflow into random energy, and to accelerate relativistic particles which can radiate a power law spectrum.

One of the interesting developments is that the $\gamma$-ray observations of GRB have become extensive enough that they are beginning to probe questions of the internal physics of the shock acceleration, the equipartition, and the radiative efficiency involved. For instance, one issue is whether the fireball, as it slows down by sweeping increasing amounts of external matter, evolves with $\Gamma \propto r^{-3 / 2}$ as expected in the adiabatic limit, or as $\Gamma \propto r^{-3}$ as expected if the remnant can tap all the kinetic energy in protons and fields and radiate it promptly (in less than a dynamic time). If the latter, the remnant evolves faster, and could reach the nonrelativistic regime sooner, even if after some time it becomes adiabatic, as it should. Physically, however, it appears difficult to see how in the cooling region (behind the shock front) the protons and fields can re-energize electrons (Mészáros, et al, 1997), even if the electrons have a cooling timescale shorter than the dynamical time, as is certainly the case early on. In fact, the optical power law of 970228 is unbroken so far, after 8 months, arguing for an early onset of the adiabatic regime, and indicating that the nonrelativistic regime has not been reached yet (which is strong indication for a cosmological distance, Wijers et al, 1997). Another observational constraint comes from the radio scintillations in 970508: this requires a relatively small size $\lesssim$ few $10^{17} \mathrm{~cm}$, after a time of several weeks. The longitudinal size is $r \sim 4(2 n+1) \Gamma_{o}^{2} c t$ where $n=(3 / 2,3)$ for an (adiabatic, radiative) remnant, but the ring structure of the remnant emitting region reduces somewhat the coefficient in front (Panaitescu \& Mészáros , 1997). The adiabatic behavior seems more in accord with observations

\section{Gamma Ray Spectra and Temporal Properties}

Two types of fireball models have been discussed, both of which produce the nonthermal spectrum via shocks occurring after the fireball has become optically thin (otherwise a nonthermal spectrum would be impossible). These involve different explanations for the typical duration of the burst, and predict different time variabilities. In the first type (a) (e.g. Rees \& Mészáros, 1992) the shocks are those caused by interaction of the gaseous fireball ejecta with an external medium. In this case the typical duration is given by the Doppler delayed arrival of the light from the beginning and end of the ejecta shell, or from the delay between surface elements within the light cone. This assumes that any "intrinsic" burst duration is shorter than the above duration (impulsive approximation). Any "intrinsic" short time variability is washed out by the fact that radiation is received from a wide light cone over which $\Gamma$ varies by at least a factor 2 . In the second type of model (b) (Rees \& Mészáros , 1994), the shocks are those which may be expected within the outflow itself, e.g. internal shocks caused by the catching up of faster portions with slower portions of the flow. These, if they occur, tend to do so at smaller radii than the previous ("external") shocks, and the duration 
is likely to be given by the intrinsic duration of the energy release (since the Doppler delayed light arrival or light cone duration is likely to be shorter than the latter). One of the two stated purposes for introducing this model was that it does specifically allow arbitrarily complicated light curves, which largely reflect any "intrinsic" variability injected at the base of the outflow (Rees \& Mészáros, 1994). This was emphasized by Sari \& Piran, 1997, who showed that external shocks in a blobby external would not be able to reproduce very jagged light curves, unless the efficiency is very low, $\lesssim 1 \%$. Nonetheless, as shown by Panaitescu \& Mészáros, if magnetic inhomogeneities are present or develop in the ejecta, and there is some pre-beaming in the comoving frame, one can get up to 5-10 peaks with good efficiency in an external shock light curve, and the spectral-temporal correlations are close to the observed values.

The nonthermal radiation spectrum of GRB is likely to be due to synchrotron or inverse Compton (IC) radiation of electrons or positrons accelerated in the optically thin shocks described above. Electrons or positron accelerated, e.g. by a Fermi mechanism, in the presence of modest magnetic fields, lead to nonthermal photon spectra similar to those observed (e.g. Mészáros, Rees \& Papathanassiou, 1994 for impulsive shock spectra, Papathanassiou \& Mészáros , 1996 for wind spectra; see also Mészáros \& Rees, 1994). Basically, two types of spectra are possible: those where the observed "break" in the $50 \mathrm{KeV}-2 \mathrm{MeV}$ range is due to the synchrotron characteristic energy, or those where it is due to the IC upscattering of a lower energy break (typically at optical energies) which itself is due to synchrotron. The latter requires smaller magnetic fields and smaller electron minimum energies $\gamma_{m}$. Above $\gamma_{m}$ shock acceleration is assumed to provide the electron power law responsible for the flattish $\nu F_{\nu}$ spectrum characteristic of bursts: an electron index $p \sim-3$ reproduces this well. The burst spectra can satisfy the X-ray paucity condition (i.e. the observation that generally $F_{x} \lesssim 10^{-2} F_{\gamma}$ ), since below the break one expects a spectrum $\nu F_{\nu} \propto \nu^{4 / 3}$ (and spectra flatter than this can be easily obtained in an inhomogeneous magnetic field, or for a spatially varying bulk Lorentz factor). In addition, one expects significant simultaneous emission at $\mathrm{GeV}$ energies, and modest but detectable simultaneous X-ray and optical emission (Mészáros \& Rees, 1993b, Mészáros, Rees \& Papathanassiou, 1994, Katz, 1994b, Papathanassiou \& Mészáros , 1996). This research has been supported in part by NASA NAG5-2857.

\section{References}

Costa, E., 1997, Proc. 4th Huntsville GRB Symp., in press; also Nature, 387, 783

Frail, D., et.al., 1997, Nature, in press

Fruchter, A. et al, 1997, IAU Circ. 6747

Goodman, J., 1997, New Astronomy, in press

Katz, J., 1994b, ApJ(Lett.), 432, L109

Katz, J. \& Piran, T., 1997, ApJ, in press

Mészáros, P \& Rees, M.J., 1993, ApJ, 405, 278

Mészáros, P, Rees, M.J. \& Papathanassiou, 1994, ApJ, 432, 181

Mészáros, P \& Rees, M.J., 1994, MNRAS, 269, L41

Mészáros, P \& Rees, M.J., 1997a, ApJ, 476, 232

Mészáros, P, Rees, M.J. \& Wijers, R., 1997, ApJ, subm. (astro-ph/9709273

Metzger, $M$ et al., 1997, Nature, 387, 878

Murakami, T., et al., 1997, Proc. Huntsville GRB Workshop, in press

Paczyński, B. \& Rhoads, J, 1993, ApJ(Lett), 418, L5

Paczyński, B., 1997, preprint

Panaitescu, A \& Mészáros, P, ApJ, in press (astro-ph/9703187)

Panaitescu, A \& Mészáros , P, ApJ(Lett), in press (astro-ph/9709284)

Pedersen, H., 1997, talk at Elba GRB Workshop

Reichart, D., 1997, ApJ, in press

Rees, M.J. \& Mészáros, P., MNRAS, 258, P41

Rees, M.J. \& Mészáros , P., ApJ(Lett), 430, L93.

Rhoads, J, 1997, preprint

Sahu, K., et al., 1997, Nature 387,476

Sari, R. \& Piran, T. 1997 (astro-ph/9701002)

Tavani, M., 1997, ApJ(Lett), 483, L87

Taylor, G.B., et al, 1997, Nature, in press

Vietri, M., 1997a, ApJ(Lett), 478, L9

Waxman, E., 1997a, ApJ(Lett), 485, L5

Waxman, E., 1997b,ApJ(Lett) subm (astro-ph/9709190)

Waxman, E., Kulkarni, S. \& Frail, D., 1997c, ApJ(Lett), subm (astro-ph/9709199)

Wijers, R.A.M.J., Rees, M.J. \& Mészáros , P., 1997, MNRAS, 288, L51 